\title{
The Effects of Scaffold Architecture and Fibrin Gel Addition on Tendon Cell Phenotype
}

\author{
K. M. Pawelec, R. J. Wardale, S. M. Best, R. E. Cameron
}

August 4, 2014

Development of tissue engineering scaffolds relies on careful selection of pore architecture and chemistry of the cellular environment. Repair of skeletal soft tissue, such as tendon, is particularly challenging, since these tissues have a relatively poor healing response. When removed from their native environment, tendon cells (tenocytes) lose their characteristic morphology and the expression of phenotypic markers. To stimulate tendon cells to recreate a healthy extracellular matrix, both architectural cues and fibrin gels have been used in the past, however, their relative effects have not been studied systematically. Within this study, a combination of collagen scaffold architecture, axial and isotropic, and fibrin gel addition was assessed, using ovine tendon-derived cells to determine the optimal strategy for controlling the proliferation and protein expression. Scaffold architecture and fibrin gel addition influenced tendon cell behavior independently in vitro. Addition of fibrin gel within a scaffold doubled cell number and increased matrix production for all architectures studied. However, scaffold architecture dictated the type of matrix produced by cells, regardless of fibrin addition. Axial scaffolds, mimicking native tendon, promoted a mature matrix, with increased tenomodulin, a marker for mature tendon cells, and decreased scleraxis, an early transcription factor for connective tissue. This study demonstrated that both architectural cues and fibrin gel addition alter cell behavior and that the combination of these signals could improve clinical performance of current tissue engineering constructs.

\section{Introduction}

Like many connective tissues, the functional properties of tendon are defined by the native extra-cellular matrix (ECM) laid down by the cells. Within healthy tissue, tendon is composed of a highly ordered matrix of collagen fibers, designed to transmit load from the muscle to the bone and to act as an elastic spring [1,2]. In addition to a dense ECM, tendon tissue contains elongated cells, thought to repair and maintain the matrix of collagen fibers [3]. 
Adult connective tissues, such as tendon, are often considered deficient in terms of healing potential, and, after injury, the restored ECM is often unaligned fibrocartilage, lacking the mechanical properties or matrix composition of undamaged tissue [4]. While it has been shown that the growth of mature tendon cells decreases with age, the response to growth factors, and the ability of cells to produce collagen Type I fibrils in vitro remains unaffected $[4,5]$. Thus, it may be a lack of environmental cues, such as reduced cell density or lack of growth factors, rather than an inability to activate the appropriate cell machinery, which causes the poor healing response [5].

Tissue engineering of tendon, to direct cell response and recreate the unique properties of healthy tissue, has focused on providing external signals to stimulate cells to produce characteristic, aligned ECM in vitro using either scaffolds or fibrin gels. An advantage of scaffolds is the ability to incorporate architectural cues, which can promote tendon cell elongation. In collagen scaffolds with an aligned pore architecture, tendon cells can be stimulated to produce an ECM more characteristic of tendon than on non-aligned scaffolds [6]. Likewise, fibrin gels, a component of the natural clotting system, can be used as support structures which allow tendon cells to align and elongate [7-9]. Unlike other gel systems, fibrin does not inhibit ECM formation, and the resulting matrix is composed of aligned collagen fibers, mimicking that in healthy tissue $[5,10]$. However, neither approach is perfect. It is difficult for cells to penetrate to the interior of collagen scaffolds, and fibrin gels are prone to instability if the dissolution of the gel is not matched by ECM production of the entrapped cells $[6,10]$.

With the addition of fibrin gels to scaffolds, tissue engineering outcomes, such as in vitro blood vessel formation and osteogenic differentiation, have been shown to improve $[11,12]$. While the advantage of adding fibrin to tissue engineering constructs is clear, the effects of combining fibrin gel and architectural cues has, to our knowledge, never been examined. Understanding the influence of each element within tissue engineering constructs is important in order to improve clinical outcomes, and we hypothesized that scaffold architecture would continue to influence tendon cell behavior, even in the presence of a fibrin gel.

Control over the incorporation of architectural cues into collagen scaffolds has been elucidated previously, and two distinct scaffold architectures were examined within this study [13, 14]. Aligned architecture, mimicking the native collagen orientation in native tendon, was compared to a homogeneous, isotropic scaffold with no preferred pore orientation. Both scaffold types were assessed with and without fibrin gel addition and compared to a fibrin only control. The biological activity of tendon tissue engineering constructs is described by two key features: their ability to stimulate cell growth and to allow cells to recreate an ECM which matches the native healthy tissue. Thus, throughout the study, cellular proliferation and metabolic activity were monitored and the composition of the matrix produced within the tissue engineering constructs was evaluated using western blotting. By understanding the independent and specific roles of collagen scaffold architecture and fibrin gel addition, better strategies can be developed 
to maximize the healing potential of adult cells.

\section{Methods}

\subsection{Scaffold Production}

Unless noted, reagents came from Sigma, UK. Suspensions of $1 \mathrm{wt} \%$ collagen were prepared by hydrating insoluble bovine Achilles tendon, Type I collagen (Sigma Aldrich) in $0.05 \mathrm{M}$ acetic acid, adjusted to $\mathrm{pH}$ 2. Slurries were homogenized (VDI 25, VWR International Ltd, UK) at 13,500 rpm for 30 minutes in an ice water bath, and centrifuged (Hermle Z300) for 5 minutes at $2500 \mathrm{rpm}$.

Specialized molds were used to alter collagen scaffold architecture. To create axial scaffolds, perspex molds with a $3 \mathrm{~mm}$ plate of copper at the base were used; the inner diameter of the molds was $8 \mathrm{~mm}$ and the height $28 \mathrm{~mm}$ [15]. The mold for isotropic scaffolds was constructed from $2 \mathrm{~mm}$ thick stainless steel with an inner diameter of $45 \mathrm{~mm} \mathrm{[15].} \mathrm{During} \mathrm{freeze-drying,} \mathrm{the}$ slurry was cooled, at a rate of $0.9{ }^{\circ} \mathrm{C} / \mathrm{min}$, to $-30{ }^{\circ} \mathrm{C}$ and held for 90 minutes, then lyophilized using a Virtis freeze drier (SP Industries, USA) at $0{ }^{\circ} \mathrm{C}$ for 20 hours under a vacuum of less than 100 mTorr. Scaffolds were cross-linked for 4 hours in a $70 \%$ ethanol solution containing a 5:2:1 ratio of $\mathrm{N}$-(3-Dimethylamino propyl)- $\mathrm{N}$ '-ethylcarbodiimide hydrochloride (EDC): $\mathrm{N}$ Hydroxysuccinimide (NHS): collagen $\mathrm{COOH}$ groups [16].

\subsection{Tendon-Derived Cell Isolation}

Ovine primary patellar tendon-derived cells were harvested by digesting minced tissues for 2 hours at $37^{\circ} \mathrm{C}$ in $0.2 \%$ collagenase (Roche) in complete media (10\% fetal bovine serum, $2 \mathrm{mM}$ glutamine, $1 \mathrm{ug} / \mathrm{ml}$ penicillin/streptomycin, $0.001 \mathrm{ug} / \mathrm{ml}$ Amphotericin, $0.01 \mathrm{ug} / \mathrm{ml}$ gentamycin). The resulting cell suspension was washed once in complete media, resuspended and plated in monolayer culture. Tendon derived cells were used between passages 4 and 5 .

\subsection{Cell Culture}

Tissue culture polystyrene (TCPS) plates were coated in SYLGARD (type 184 silicone elastomer, Dow Chemical) and set overnight at $60^{\circ} \mathrm{C}$ [7]. For sterilization, plates were soaked overnight in $70 \%$ ethanol. Scaffolds were pinned to the SYLGARD with minutiens insect pins (0.1 mm diameter, Fine Science Tools GmbH, Germany), one on either side of each scaffold and sterilized by immersion in $70 \%$ ethanol for one hour, then washing twice in 1x phosphate buffered saline (PBS). Before seeding cells, PBS was replaced with either media, for samples without fibrin, or with PBS, for samples with fibrin. 
Immediately before seeding cells, scaffolds were blotted in situ with sterilized filter paper (3MM, Whatman). Fibrin gels were prepared by adding $1 \%$ fibrin and aprotinin $(0.15 \mathrm{U} / \mathrm{ml})$ to PBS. Scaffolds were seeded with $1 \times 10^{6}$ tendon cells in $75 \mu$ of either media or fibrin. Scaffolds without fibrin gel were incubated $\left(5 \% \mathrm{CO}_{2}, 37{ }^{\circ} \mathrm{C}\right)$ for 2 hours before flooding the well with complete media. For scaffolds with fibrin gels, immediately after addition of suspension, scaffolds were immersed in $300 \mu \mathrm{l}$ of $0.4 \mathrm{U} / \mathrm{ml}$ thrombin and allowed to set for 10 minutes. Excess thrombin was removed and replaced with complete media. Empty, control scaffolds were treated as other scaffolds, but with media or fibrin which did not contain cells. The fibrin control, adapted from Kapacee et al [7], was constructed by seeding $1 \times 10^{6}$ cells in $75 \mu$ l of fibrin across a $10 \mathrm{~mm}$ gap between minutiens pins. The fibrin control was gelled in $75 \mu \mathrm{l}$ of $0.4 \mathrm{U} / \mathrm{ml}$ thrombin. To compare to the fibrin control, all scaffolds were $10 \mathrm{~mm}$ in length, with a cross-section of $5 \times 5 \mathrm{~mm}$. The samples groups were: axial $(\mathrm{AX})$, axial+fibrin $(\mathrm{AX}(\mathrm{F}+))$, isotropic (ISO), isotropic+fibrin (ISO(F+)), and fibrin (F+).

\subsection{Cell Metabolic Assay}

An alamarBlue ${ }^{\circledR}$ assay (Invitrogen, UK) was used to test cell metabolism on days 3, 10, and 17. Samples were incubated in media containing $10 \%$ alamarBlue. Cells were incubated for 4 hours at $37{ }^{\circ} \mathrm{C}$. The fluorescence of the cell supernatant was read using a Fluorstar optima plate reader (BMG Labtech).

\subsection{Cell Number}

The number of cells attached to the constructs was measured using DNA concentration, which was evaluated via the Hoechst assay after 7, 14 and 21 days [17]. Cells and scaffolds were digested at $60^{\circ} \mathrm{C}$ overnight in papain buffer [18]. Briefly, $10 \mu \mathrm{l}$ of papain digest was added to Hoechst dye (Bisbenzimide $\mathrm{H}$ ) for a final dye concentration of $0.1 \mu \mathrm{g} / \mathrm{ml}$. Fluorescence was measured on a Fluorstar Optima plate reader (360nm Ex, 460nm Em).

\subsection{Matrix Production}

Protein expression at 21 days was analyzed using SDS-PAGE and Western blotting. Constructs were incubated overnight at $4{ }^{\circ} \mathrm{C}$ in cell lysis buffer (Invitrogen) and mixed with sodium dodecyl-sulphate (SDS) sample buffer for a final concentration of 1x, before incubation at 60 ${ }^{\circ} \mathrm{C}$ for 20 minutes. Standards were prepared from native ovine patellar tendon tissue, which was snap frozen in liquid nitrogen, milled using a dismembrator (B Braun Biotech International $\mathrm{GmbH})$, and suspended in cell lysis buffer $(100 \mathrm{mg} / \mathrm{ml})$. 
A Novex assembly with commercially available 4-20\% Tris-glycine gels (Invitrogen) was used. Proteins were separated (125 V for 2 hours), and were transferred to a membrane (Immobilon PVDF, Millipore) for Western blotting. Tendon constructs were probed for fibronectin (SC9068, Santa Cruz Biotech), tenascin-C (ab82449 Abcam), tenomodulin (ab81328 Abcam), scleraxis (OAAB00449 Aviva Sys Biology), and collagen Type I (Rockland). Peroxidase secondary antibodies were used throughout.

After immunoprobing, membranes were developed using ECL plus reagents (Amersham), per manufacturer's instructions. Signal strength was assessed using a densitometer (BioRad GS800). All data is represented as the fold change from the axial scaffold without fibrin. Within each sub-figure, all of the lanes are from the same gel; where lanes have been moved for clarity, it has been clearly marked. Data was normalized by DNA concentration due to interference of serum proteins with protein normalization (data not shown).

\subsection{Immunohistochemical Staining}

Constructs were frozen in $\mathrm{OCT}^{\mathrm{TM}}$ Compound (Tissue Tek) and sectioned into $10 \mu \mathrm{m}$ slices with a microtome (Bright, UK). Tissue sections were fixed in 1:1 acetone-methanol, and washed in PBS-0.1\% Tween. Sections were incubated with a fibronectin antibody (SC-9068, Santa cruz Biotech) at 1:200 dilution in PBS-tween for 4 hours, and placed in FTIC-conjugated secondary antibodies for 1 hour. Sections were mounted with Vectashield+DAPI (Vector Labs).

\subsection{Scanning Electron Microscopy}

All scanning electron microscopy images were taken using a JEOL 820, with a tungsten source, operated at $10 \mathrm{kV}$. For images of cells on scaffolds, cells were fixed for 1 hour in $4 \%$ paraformaldehyde and samples were dried by soaking for 20 minutes in ethanol dilutions, modified from [19]. The ethanol dilutions were as follows: $25 \%, 30 \%, 50 \%, 70 \%, 80 \%$, and $90 \%$ ethanol. Scaffolds were then washed twice, in $100 \%$ ethanol, and transferred to a 50/50 v/v mixture of ethanol and Hexamethyldi-silazane (HDMS), before replacing the solution with 100\% HDMS. Samples were allowed to air dry overnight. All sections of the collagen scaffolds were sputter coated with platinum, at a current of $40 \mathrm{~mA}$ prior to imaging.

\subsection{Statistics}

Metabolic activity had a sample size of 8 , and 3 replicates were used for cell number studies. All western blots were run with three replicates. Groups were compared with ANOVA followed by a post-hoc Tukey HSD test, with a 95\% confidence interval. All data is presented with standard deviations. 

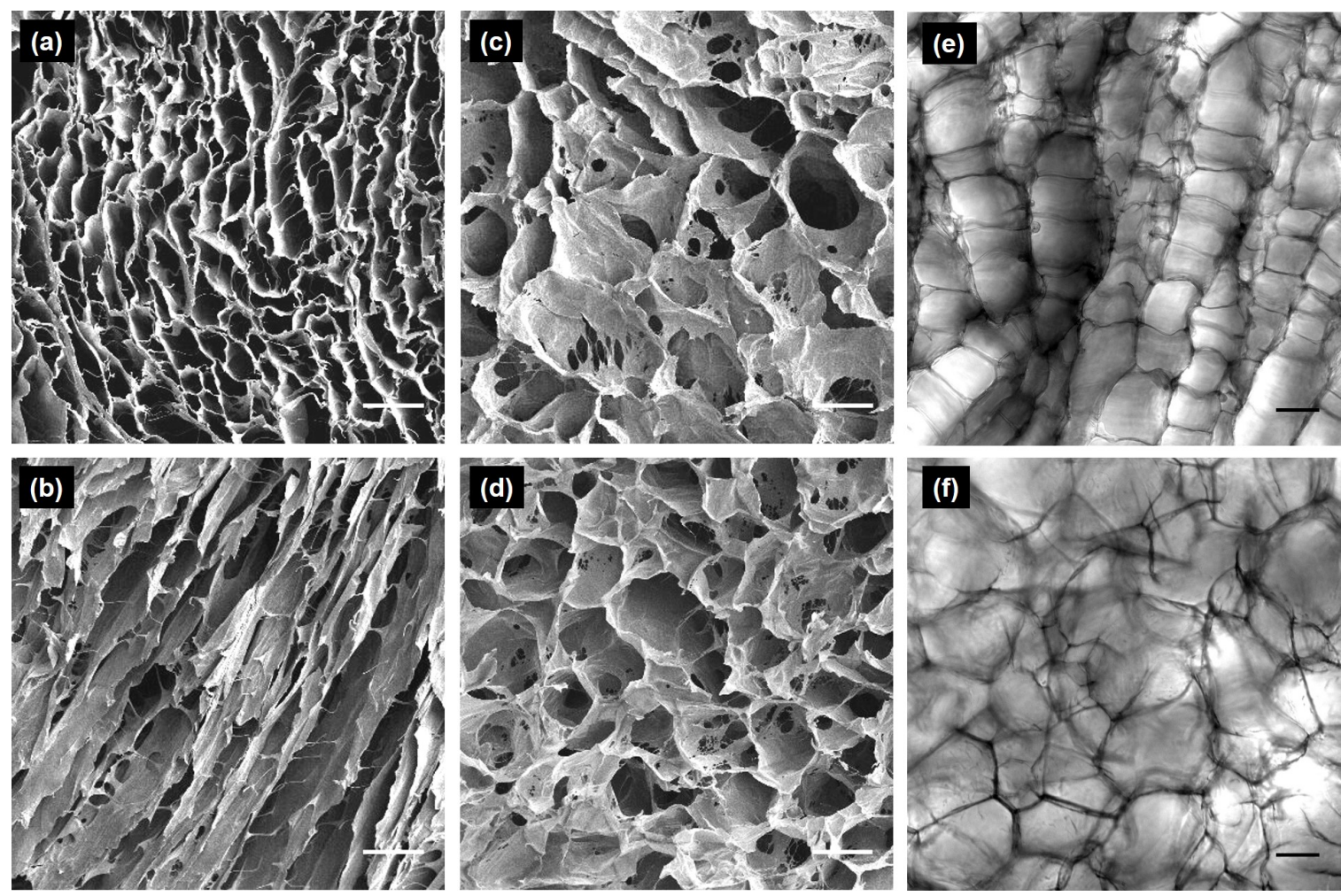

Figure 1: Structure differed markedly between axial and isotropic scaffolds. Axial scaffolds had no preferred orientation in the (a) transverse direction, but consisted of (b) aligned pores in the longitudinal direction, running from the scaffold base to the top. Isotropic scaffolds were had no preferred orientation in any direction, but had the same structure in the (c) longitudinal and (d) transverse direction. Upon hydration, (e) axial scaffolds and (f) isotropic scaffolds had a similar pore size. Scale bar is $100 \mu \mathrm{m}$.

\section{Results}

\subsection{Scaffold Architecture}

Two different structures were formed: axial and isotropic scaffolds (Fig. 1). Axial scaffolds, as has been demonstrated previously in the literature, consisted of aligned pores in the longitudinal direction (Fig. 1a), while in the transverse plane, there was no predominant directionality of the pores (Fig. 1b) [6]. Isotropic scaffolds consisted of purely equiaxed pores with no preferred orientation (Fig. 1c, d). In the hydrated state, the pore size of the scaffolds appeared similar (Fig. 1e, f). Both scaffold types have a pore size of between $100-130 \mu \mathrm{m}$, as shown in a previous study [15]. Tendon cells were found to adhere on all scaffold constructs tested, regardless of scaffold structure (Fig. 2). With the addition of fibrin gels to collagen scaffolds, cell number increased visibly, and tendon cells appeared more well spread on the cross-linked collagen surface (Fig. 2c, d). 

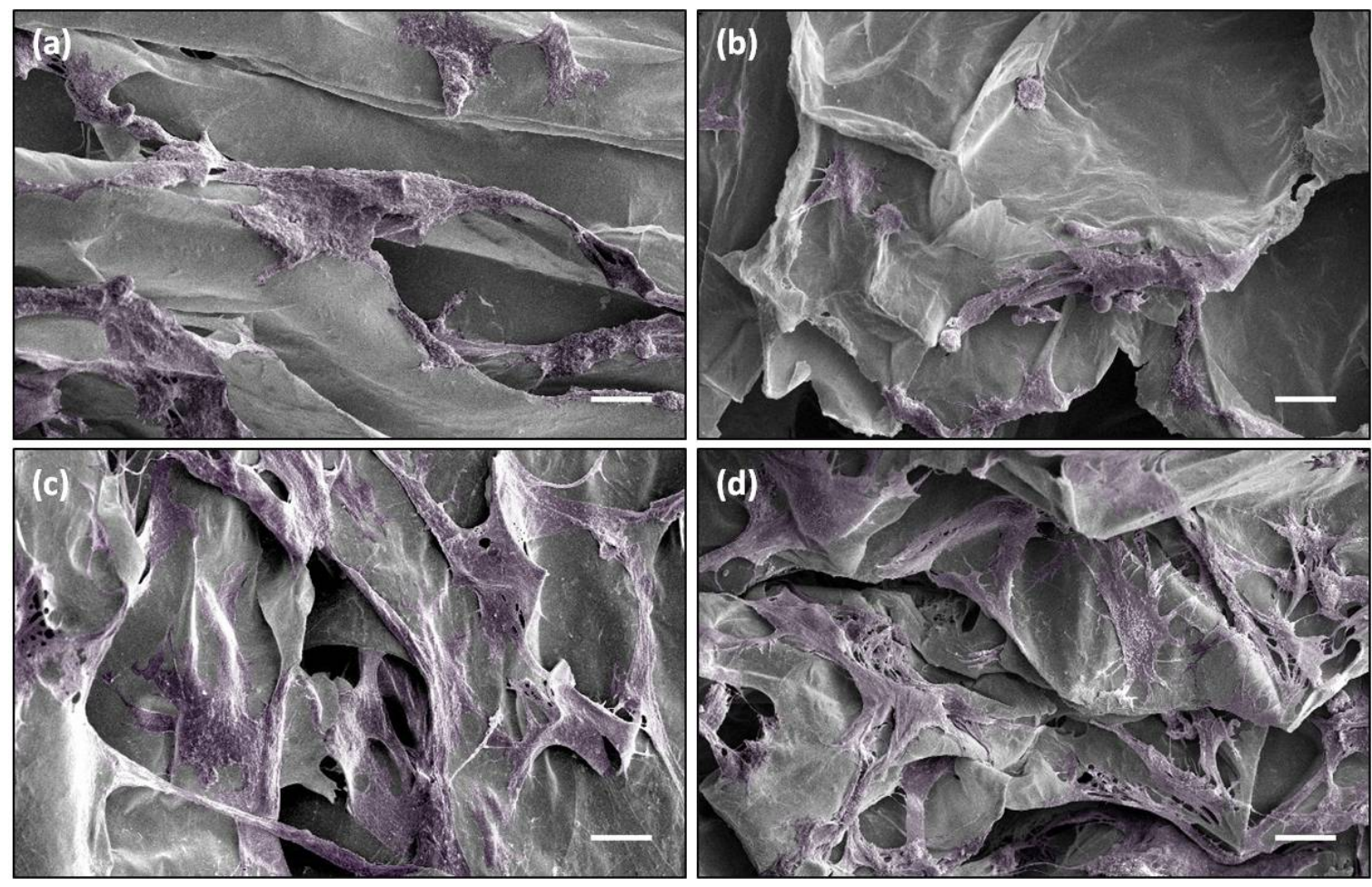

Figure 2: Tendon cells could adhere to all scaffold constructs: (a) axial scaffold, (b) isotropic scaffold, (c) axial scaffold with fibrin, (d) isotropic scaffold with fibrin. With fibrin gel addition, the cells appeared well spread. Scale bar is $20 \mu \mathrm{m}$.

\subsection{Cellular Metabolic Activity and Number}

Cell metabolic activity increased significantly in all scaffolds over a three week period (Fig. 3). After 21 days, there were no significant differences between cell metabolic activity on isotropic or axial scaffolds. While scaffold architecture produced no significant changes in metabolic activity, fibrin addition to scaffolds increased cell metabolic activity above that of scaffolds alone (Fig. 3a). A significant decrease was observed in the cell metabolic activity in the fibrin control (Fig. 3a).

Cell number remained relatively constant over the culture period. There was no significant difference between cell number on axial and isotropic architectures after three weeks, even in the presence of a fibrin gel. However, the addition of a fibrin gel to scaffolds increased sell number significantly, even above the fibrin control (Fig. 3b).

\subsection{Extra Cellular Matrix Production}

The final outcome of the constructs was assessed via Western blotting after 21 days in culture and normalized via DNA concentration to determine relative matrix production independent of cell proliferation within the constructs. Constructs were assessed for fibronectin, scleraxis, and tenomodulin, which are accepted markers of tendon phenotype [20]. Tenascin-C expression 

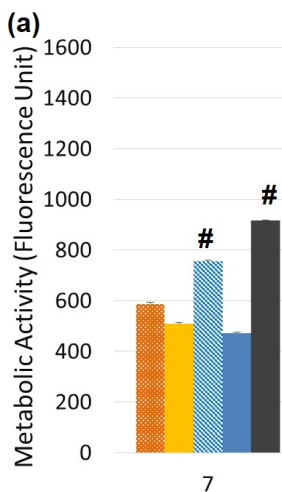

7

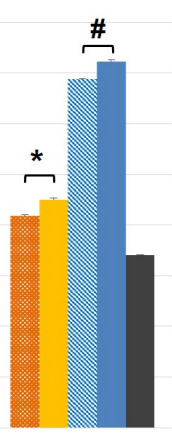

21

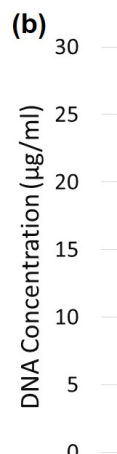

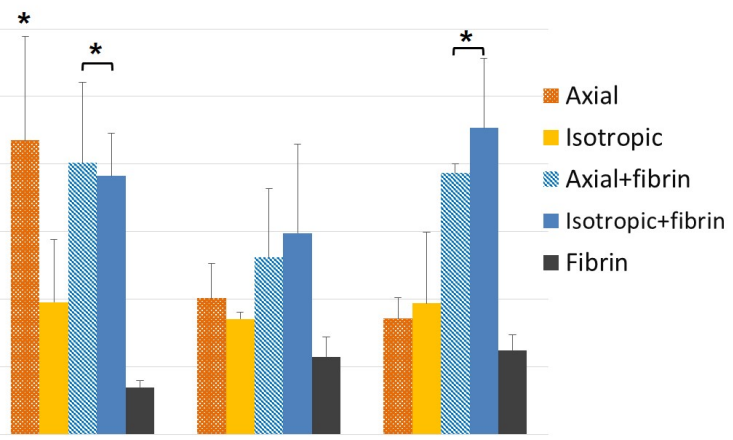

21

Figure 3: The effect of scaffold architecture and fibrin gel addition on (a) metabolic activity as measured by the alamarBlue assay and (b) cell number evaluated using the Hoechst assay. * Significantly greater than the fibrin control $(\mathrm{p}<0.05)$. \# Significantly different from all other sample types $(\mathrm{p}<0.05)$.

was also evaluated, but it was found that none of the constructs had strong expression of the protein, so further quantification was not performed. In addition to tendon specific phenotype markers, fibronectin was used as a general marker of cellular attachment to scaffold structures. All protein expression was reported as a fold change from axial scaffolds without fibrin.

\section{Fibronectin}

Fibronectin was found to be specific to cellular activity and was not expressed in empty scaffolds (Figure 4a). Fibronectin is known to be dimeric with a calculated molecular weight of $220 \mathrm{kDa}$ (monomer) [21]. Faint bands at $220 \mathrm{kDa}$ were visible with a stronger band at roughly $440 \mathrm{kDa}$, suggesting that most of the fibronectin was dimerized.

The amount of fibronectin appeared to vary with scaffold architecture. Cells on isotropic scaffolds produced greater amounts of fibronectin than on axial scaffolds. This trend was true with and without fibrin gel addition. Immunohistochemistry on axial and isotropic scaffolds revealed that the majority of fibronectin within the matrix was located along scaffold edges, (Fig. 5). The addition of fibrin gel to scaffolds resulted in a lower fibronectin expression (Fig. $4 c)$.

\section{Collagen Type I}

Even though the scaffolds themselves were made of collagen Type I fibers, the empty scaffolds had no visible signal when the antibody was tested (Fig. 4d), likely because the collagen of the scaffold was not soluble in the lysis buffer used to extract the ECM, being made from acid insoluble collagen which has mature cross-links [22]. Bands, corresponding to the alpha 1(I) and alpha 2(I) chains of collagen, were seen at $139 \mathrm{kDa}$ and $129 \mathrm{kDa}$, respectively [23]. The 
(a) $\mathrm{AX}$ ISO $\mathrm{AX}(\mathrm{F}+) \mathrm{ISO}(\mathrm{F}+)(\mathrm{F}+$

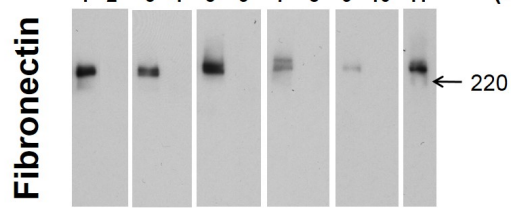

(d) $\mathrm{AX}$ ISO $\mathrm{AX}(\mathrm{F}+) \mid \mathrm{SO}(\mathrm{F}+\mathrm{X}+\mathrm{F}+)$
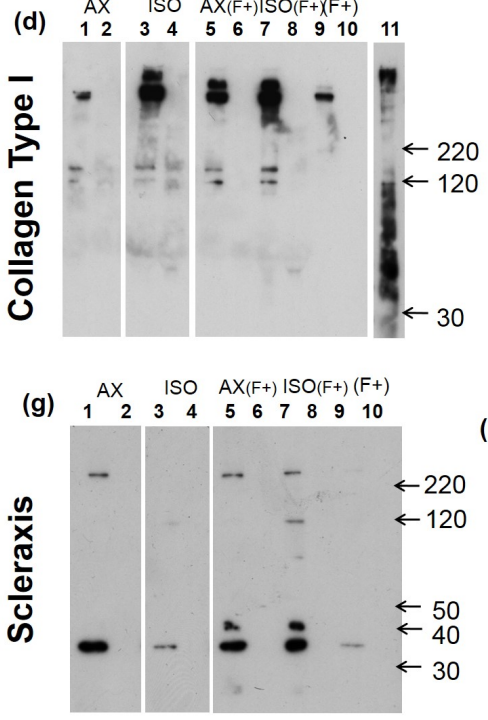

(j) $\mathrm{AX}$ iso $\mathrm{AX}(\mathrm{F}+) \mathrm{SO}(\mathrm{F}+)(\mathrm{F}+)$

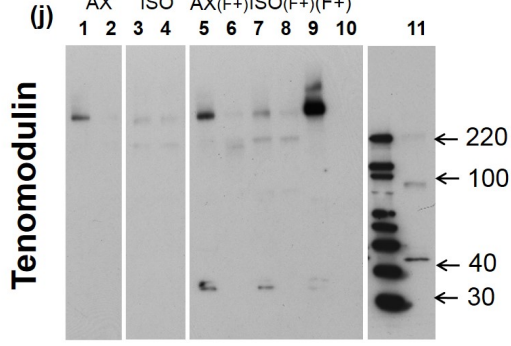

(h) (b)

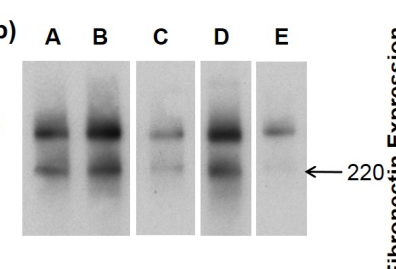

(e)
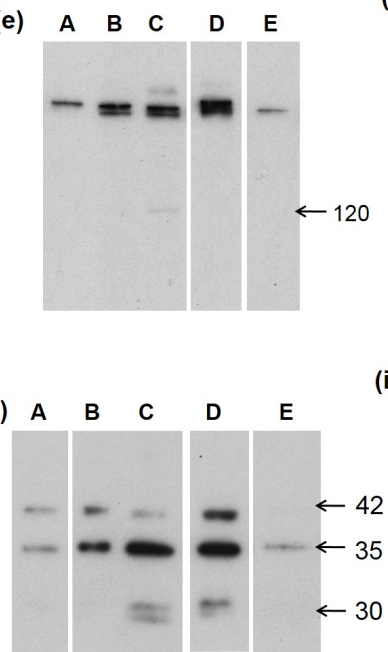

(k)

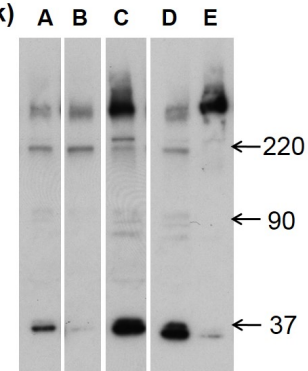

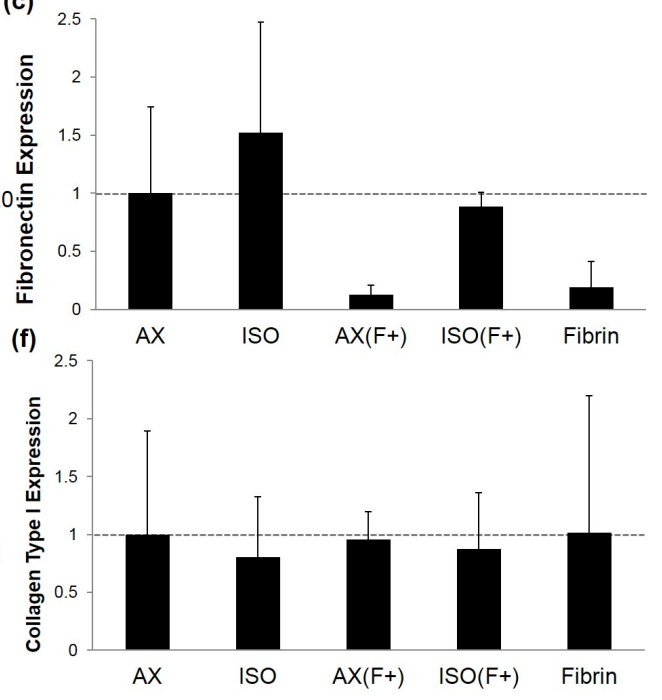

(c)

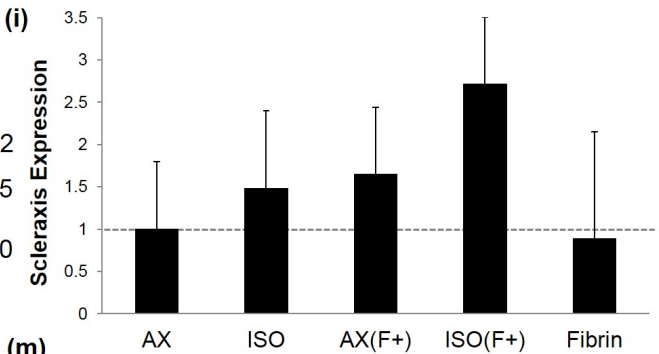

(m)

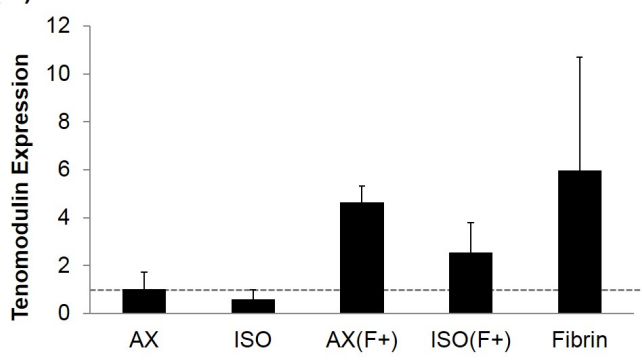

Figure 4: Protein expression differed with architecture and addition of fibrin for (a-c) fibronectin, (d-f) collagen Type I, (g-i) scleraxis, and (j-m) tenomodulin. (a, d, g, j) Protein expression was specific to cells. 1: $A X ; 2$ : empty $A X ; \mathbf{3}: I S O ;$ 4: empty ISO; 5: $A X(F+) ; \mathbf{6}$ : empty $A X(F+)$; 7: $I S O(F+) ; 8:$ empty $I S O(F+) ; 9:(F+) ; 10:$ empty $(F+) ; 11:$ tendon tissue. (b, e, h, k) Protein expression varied with the sample type. A: $A X ; \mathbf{B}: I S O ; \mathbf{C}: A X(F+)$; D: $I S O(F+)$; E: fibrin. (c, f, i, m) Protein expression presented as the fold change from AX scaffold, measured via densitometry. 

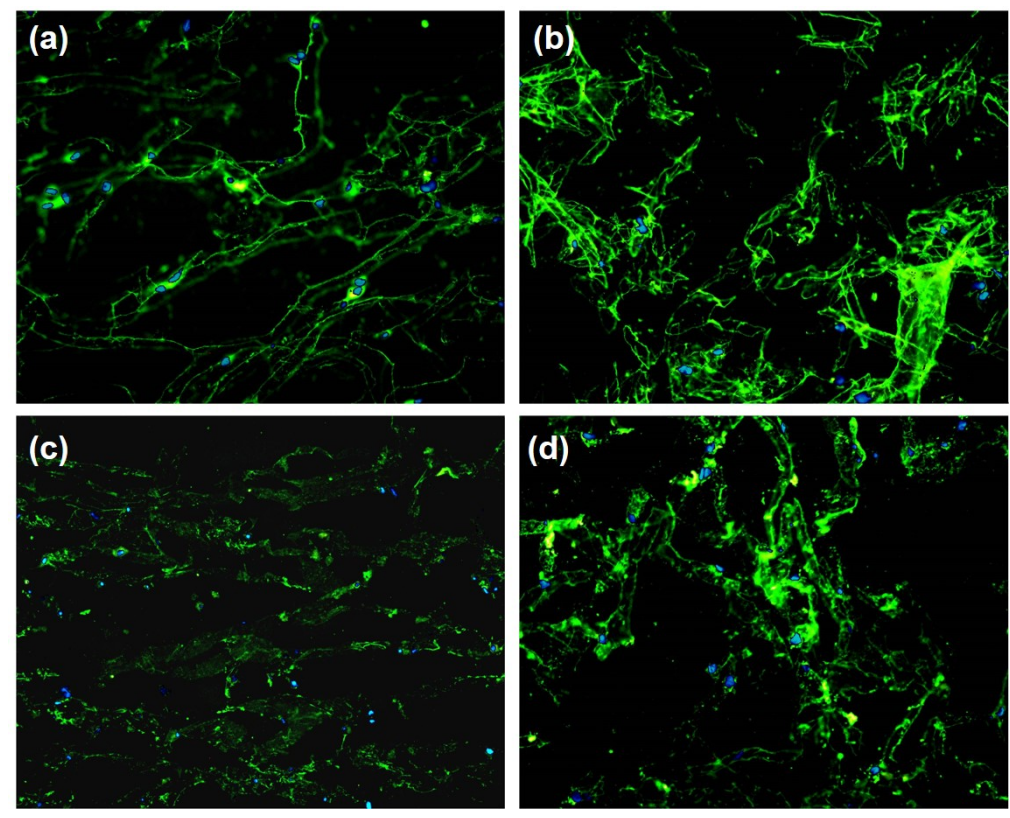

Figure 5: Immunohistochemistry staining of tendon cells for fibronectin in (a) axial scaffolds, (b) isotropic scaffolds, (c) axial scaffolds + fibrin, and (d) isotropic scaffolds + fibrin. Blue is DAPI nuclear stain, green is fibronectin. Magnification is 20x.

bands above $220 \mathrm{kDa}$, multimers of the alpha chains, produced a much stronger signal. Native tendon tissue had a smear of low molecular weight fragments, probably due to degradation of the collagen during processing (Fig 4d, lane 11). Tendon cells formed collagen Type I in all scaffold orientations and culture systems (Fig. 4e). There was no difference in expression between axial or isotropic scaffolds, or even with the addition of a fibrin gel once the protein expression was normalized by DNA content (Fig 4f).

\section{Scleraxis}

Blots of scleraxis showed bands at 30,35 , and $42 \mathrm{kDa}$, with an occasional unreducible band at higher molecular weights (Fig. 4g). As a transcription factor associated with connective tissue, scleraxis has a predicted size of roughly 22 - $28 \mathrm{kDa}[20,24]$. However, within this study, scleraxis was found in unreducible bands at 37 and $42 \mathrm{kDa}$, which are not reported in literature. While the antibody is well characterized and commercially manufactured, it is not known whether isoforms of scleraxis exist, or the precise mechanism of scleraxis regulation, especially in ovine models $[25,26]$. No scleraxis expression was detected from native tendon tissue at any point, even though adult tissues have been shown to express scleraxis mRNA throughout life and that gene expression can be induced in adults with exercise [27,28]. However, these studies have been based on gene expression levels rather than protein expression, and it is possible that the protein levels produced in the adult tissue are below detection limits.

Scleraxis signal came from cellular activity (Fig. 4g), but, interestingly, no signal for scleraxis was ever seen in native tendon tissue, although cells expressed scleraxis in all tendon constructs. 
Cells on isotropic scaffolds had a greater scleraxis expression than axial scaffolds(Fig. 4i). This effect of architecture remained, even with the addition of fibrin. However, the addition of fibrin to scaffolds increased the scleraxis expression within constructs (Fig. 4i). The $30 \mathrm{kDa}$ band, reported in literature, only appeared when cells were cultured in scaffolds with fibrin (Fig. 4h). While the mean expression level of scleraxis was lower in fibrin gel than in any other construct, the variability was high.

\section{Tenomodulin}

In native tendon tissue, tenomodulin bands were found at 35, 95, and $220 \mathrm{kDa}$ (Fig. 4j). Within scaffolds, most tenomodulin signal was detected in two bands around $40 \mathrm{kDa}$, and in another doublet at $220 \mathrm{kDa}$ and higher. Tenomodulin expression was higher on axial scaffolds than on isotropic scaffolds (Fig. 4m), which was clearly visible after fibrin gel addition. The addition of fibrin to scaffolds caused an increase in tenomodulin expression. Fibrin gel on its own appeared to stimulate tenomodulin expression, however, the degree of up-regulation was highly variable between samples. Bands were detected at approximately the predicted molecular weight of tenomodulin, but there were also bands at higher molecular weights $[24,29,30]$. Glycosylation of tenomodulin can shift the molecular weight slightly, from 37 to $45 \mathrm{kDa}$, but no higher molecular weight bands have been reported in literature and reduction of the samples did not eliminate the bands [29].

\section{Discussion}

\subsection{Scaffold Architecture}

By tailoring tissue engineering constructs with a diverse range of signals, cellular response can be manipulated to allow regeneration of adult tissues. For tendon, a tissue with inherent structure, architectural cues have been shown to help maintain a characteristic phenotype in vitro $[31,32]$. While scaffold architecture has recently been shown to promote tendon cell phenotype, fibrin gels have also been used as models for tendon tissue engineering as they encourage cellular proliferation and ECM production [7]. The combination of scaffolds and fibrin gel have had encouraging results for in vitro systems, however, no systematic study has yet been done which examines the biological influence of scaffold architecture and the addition of fibrin gels.

In this study, scaffold architecture was controlled by the mold used for ice-templating $[6,15]$. Isotropic scaffolds were formed when ice nucleation occurred throughout the slurry volume, while axial scaffolds resulted when crystals nucleated only at the base of the mold and grew upwards [13]. Ice-templated, axial scaffolds have been shown previously in the literature to 
be significantly aligned in the longitudinal plane, and to promote an elongated tendon cell morphology compared to isotropic scaffolds [6]. Although in the dry state, the scaffold pore size appeared different, when hydrated, the scaffolds expanded to have pore sizes in the range of $100-130 \mu \mathrm{m}$ [15]. It is expected that the direction of cellular migration, and perhaps the speed of migration, might be altered by the pore structure. As the pore size was not significantly different between axial and isotropic scaffolds, the structure should not have been a barrier to cell infiltration during the study. Altering the pore structure and the addition of fibrin gel could also change the mechanical properties of the scaffold and potentially lead to large contraction of scaffold constructs, triggering a loss of tendon cell viability [33]. However, no scaffold construct contracted in any direction macroscopically, due to the relative density of the scaffolds and the anchoring of the constructs during culture. Thus, all constructs were able to support tendon cell proliferation and matrix production.

\subsection{Evaluating Cell Proliferation and Metabolic Activity}

Cell metabolic activity was not affected by scaffold architecture, but was increased significantly with the addition of fibrin gel into scaffolds. While diffusion of the dye may be altered by fibrin gel addition, causing an underestimation of the true metabolic activity of the tendon cells, the results were still significantly higher on scaffolds with fibrin addition than without. The increase in metabolic activity, corresponding to increased reduction of alamarBlue $®$, can indicate either increased cellular proliferation or ECM synthesis, both of which require large amounts of metabolic energy [34]. The decrease in metabolic activity in the fibrin control over 21 days corresponded to a tendency to dissolve over the three week period, as has been noted in literature [10]. It seems probable that the dissolution of the gel was not matched by ECM production, resulting in a decrease in cell number and cell metabolic activity.

The cell number within the scaffold constructs remained constant, over the culture period, suggesting that cell death and proliferation were balanced. In some cases, there was a decrease in cell number over the course of the study, a phenomenon which has been reported previously in the literature [6]. The variability in cell number between constructs may be due to greater initial cell attachment. The steady state in cell number suggests that changes in metabolic activity may have been related to increased matrix production or remodeling in scaffolds with fibrin gel.

\subsection{Relative Contributions of Architecture and Fibrin Gel}

A major aspect of tissue engineering is to encourage cells to reproduce a functional native matrix, an ability which tendon cells can lose during in vitro culture [27,35], and therefore it is important to characterize the ECM produced by the cells. Although no precise tendon 
phenotype has been defined in the literature, all of the proteins examined have been cited as markers of tendon phenotype [20,27]. Tendon cells in all of the constructs expressed collagen Type I, scleraxis and tenomodulin, suggesting that the tendon cell phenotype was maintained during the study. However, the tissue engineering construct affected the relative amount of protein marker expression.

Overall, scaffold architecture had the largest effect on protein expression, but did not influence tendon cell number or metabolic activity. Both isotropic and axial scaffold architectures maintained tendon cell number over three weeks. Protein expression, however, was sensitive to scaffold architecture, both with and without a fibrin gel. Tendon cells within isotropic scaffolds produced higher amounts of both fibronectin and scleraxis than axial scaffolds. In contrast, axial architecture stimulated tenomodulin production, which is thought to be a marker for a mature tendon cells phenotype [20,29]. Isotropic scaffolds may promote a less mature phenotype, as scleraxis is associated with the early stages of development in connective tissue, and protein expression was not found in mature native tendon within the current study [20,36].

Unlike scaffold architecture, addition of fibrin had a profound effect on cellular metabolism and number, resulting in a significant increase in both. In addition, addition of a fibrin gel influenced protein expression within scaffolds, causing a decrease in fibronectin expression regardless of scaffold architecture. Given the supportive matrix which fibrin forms around cells, it is likely that the cells could easily move within the gel without producing large amounts of adhesive proteins, explaining the reduction of expression seen in Western blotting. However, the appearance of fibronectin along the walls of scaffolds, seen in Figure 5, suggested that the cells remained close to the pore walls, rather than moving within the pore space.

The addition of a fibrin gel to scaffolds also increased the expression of both scleraxis and tenomodulin. Fibrin gels have been noted in literature to cause increased ECM production [10], consistent with the findings of the current study. While expression was up-regulated regardless of scaffold architecture, the effects of scaffold architecture were still present. Within the fibrin gel controls, protein expression data was affected by gel degradation over the culture period. The gel degradation added variability to protein expression data, and highlighted the improved stability of the construct with a scaffold. This has been noted in the literature, where it has been demonstrated that scaffolds and fibrin gels stimulate the greatest healing response when used together $[11,12,37]$.

Unlike the other proteins tested, collagen Type I content remained stable regardless of scaffold architecture or fibrin gel addition. Gene expression differences have been noted as the culture system moves from 2D to 3D, and with the change in environmental cues, such as the weight percent of collagen in scaffolds $[27,33]$. The results from the present study indicate that collagen content is not affected by the presence or absence of a fibrin gel, or by the presence of a supporting scaffold. Cell number appeared to be the only determiner of collagen Type I expression. Therefore, constructs which can support higher cell numbers also have an increased 
collagen Type I within the matrix, which is essential to the regeneration of tendon tissue.

Throughout the study, it was seen that architecture played an important role on cell morphology, even in the presence of a fibrin gel. The fact that trends in protein expression were consistent with scaffold architecture, despite fibrin gel addition, suggests that the scaffold architecture plays a key role in regulating tendon phenotype and function. The ability of the scaffold structure to alter cellular morphology most likely drove the effects of scaffold architecture. Axial scaffolds appeared to promote a more mature tendon cell phenotype than isotropic scaffolds, and, indeed, other studies within tendon tissue engineering, have reported a strong link between tendon cell elongation and phenotypic gene expression $[32,38]$. It has also been found that elongated tendon cells tend to produce collagen fibers which are also aligned like native tissue, and able to withstand much higher mechanical loading than unaligned collagen networks [7]. This suggests that for tendon tissue engineering, a scaffold with axial architecture offers an advantage over isotropic scaffolds.

Addition of a fibrin gel clearly increased cell metabolic activity and cell number. Even though fibrin gel caused an up-regulation of tendon matrix production, addition of the gel did not alter the phenotype due to scaffold architecture. Thus, the increase in cell number promoted by fibrin gel is independent of protein expression of cells, which is influenced by the scaffold architecture. When used together, combinations of fibrin gel addition and scaffold architecture can boost cell number and encourage the production of native extra cellular matrix.

\section{Conclusions}

Tenocytes have been cultured in scaffolds with controlled pore architectures, ranging from isotropic to aligned, and the effects of the addition of a fibrin gel has been studied. The cell proliferation and protein expression of tendon cells was studied over three weeks, and it was found that both architectural cues and fibrin gel addition regulate tendon cell response independently. While fibrin gel addition increased matrix production and led to a higher metabolic activity and cell proliferation, the cellular phenotype appeared to be controlled by scaffold architecture. Tendon cells in scaffolds with aligned pore structures, which mimicked the native organization of Type I collagen, expressed higher amounts of tenomodulin, which is linked to a mature tendon phenotype. Isotropic scaffolds promoted fibronectin and scleraxis expression. Fibrin has a positive impact on tendon cell growth and matrix synthesis, and scaffold architecture is important for maintaining an elongated tendon cell and thus controlling the cell phenotype. Scaffold architecture and fibrin gel have an additive effect on the tissue engineering construct and it is recommended that future constructs designed for tendon repair combine these strategies. 


\section{Acknowledgments}

The authors gratefully acknowledge the financial support of the Gates Cambridge Trust, the ERC Advanced Grant 320598 3D-E, and the Technology Strategy Board UK. Special thanks to Nigel Loveridge for help with the statistical analysis and Natalia Davidenko for supplying the freeze drying molds.

\section{References}

[1] J. Kastelic, A. Galeski, and E. Baer, "Multicomposite structure of tendon," Connective Tissue Research 6(1), pp. 11-23, 1978.

[2] A. Biewener, Tendons and Ligaments: Structure, Mechanical Behavior and Biological Function, book section 10, pp. 269-284. Springer, New York, 2008.

[3] D. Amiel, C. Frank, F. Harwood, J. Fronek, and W. Akeson, "Tendons and ligaments a morphological and biochemical comparison," Journal of Orthopaedic Research 1(3), pp. 257-265, 1984.

[4] F. Klatte-Schulz, S. Pauly, M. Scheibel, S. Greiner, C. Gerhardt, G. Schmidmaier, and B. Wildemann, "Influence of age on the cell biological characteristics and the stimulation potential of male human tenocyte-like cells," European Cells \& Materials 24, pp. 74-89, 2012.

[5] M. Bayer, C.-Y. C. Yeung, K. E. Kadler, K. Qvortrup, K. Baar, R. B. Svensson, S. P. Magnusson, M. Krogsgaard, M. Koch, and M. Kjaer, "The initiation of embryonic-like collagen fibrillogenesis by adult human tendon fibroblasts when cultured under tension," Biomaterials 31(18), pp. 4889-4897, 2010.

[6] S. R. Caliari and B. A. C. Harley, "The effect of anisotropic collagen-gag scaffolds and growth factor supplementation on tendon cell recruitment, alignment, and metabolic activity," Biomaterials 32(23), pp. 5330-5340, 2011.

[7] Z. Kapacce, S. H. Richardson, Y. Lu, T. Starborg, D. F. Holmes, K. Baar, and K. E. Kadler, "Tension is required for fibripositor formation," Matrix Biology 27(4), pp. 371-375, 2008.

[8] N. S. Kalson, D. F. Holmes, Z. Kapacee, I. Otermin, Y. Lu, R. A. Ennos, E. G. CantyLaird, and K. E. Kadler, "An experimental model for studying the biomechanics of embryonic tendon: Evidence that the development of mechanical properties depends on the actinomyosin machinery," Matrix Biology 29(8), pp. 678-689, 2010. 
[9] M. E. Smithmyer, L. A. Sawickia, and A. M. Kloxin, "Hydrogel scaffolds as in vitro models to study fibroblast activation in wound healing and disease," Biomaterials Science 2 , pp. 634-650, 2014.

[10] J. J. Ross and R. Tranquillo, "Ecm gene expression correlates with in vitro tissue growth and development in fibrin gel remodeled by neonatal smooth muscle cells," Matrix Biology 22(6), pp. 477-490, 2003.

[11] A. Lesman, J. Koffler, R. Atlas, Y. J. Blinder, Z. Kam, and S. Levenberg, "Engineering vessel-like networks within multicellular fibrin-based constructs," Biomaterials 32(31), pp. 7856-7869, 2011.

[12] N. Lohse, J. Schulz, and H. Schliephake, "Effect of fibrin on osteogenic differentiation and vegf expression of bone marrow stromal cells in mineralized scaffolds: a threedimensional analysis," European Cells \& Materials 23, pp. 413-424, 2012.

[13] K. M. Pawelec, A. Husmann, S. M. Best, and R. E. Cameron, "Understanding anisotropy and architecture in ice-templated biopolymer scaffolds," Materials Science \& Engineering C 37, pp. 141-147, 2014.

[14] K. M. Pawelec, A. Husmann, S. M. Best, and R. E. Cameron, "A design protocol for tailoring ice-templated scaffold structure," Journal of the Royal Society Interface 11, p. 20130958, 2014.

[15] N. Davidenko, T. Gibb, C. Schuster, S. M. Best, J. J. Campbell, C. J. Watson, and R. E. Cameron, "Biomimetic collagen scaffolds with anisotropic pore architecture," Acta Biomaterialia 8(2), pp. 667-676, 2012.

[16] L. Damink, P. J. Dijkstra, M. J. A. vanLuyn, P. B. vanWachem, P. Nieuwenhuis, and J. Feijen, "Cross-linking of dermal sheep collagen using a water-soluble carbodiimide," Biomaterials 17(8), pp. 765-773, 1996.

[17] Y.-J. Kim, R. L. Sah, J.-Y. H. Doong, and A. J. Grodzinsky, "Fluorometric assay of dna in cartilage explants using hoechst 33258," Analytical Biochemistry 174(1), pp. 168 - 176, 1988.

[18] R. Farndale, D. J. Buttle, and A. J. Barrett, "Improved quantitation and discrimination of sulphated glycosaminoglycans by use of dimethylmehtylene blue," Biochem Biophys Acta. 883(2), pp. 173 - 177, 1986.

[19] J. Venugopal, L. L. Ma, T. Yong, and S. Ramakrishna, "In vitro study of smooth muscle cells on polycaprolactone and collagen nanofibrous matrices.," Cell Biology International 29, pp. 861-867, 2005. 
[20] C. Shukunami, A. Takimoto, M. Oro, and Y. Hiraki, "Scleraxis positively regulates the expression of tenomodulin, a differentiation marker of tenocytes," Developmental Biology 298(1), pp. 234-247, 2006.

[21] E. Pearlstein, L. I. Gold, and A. Garciapardo, "Fibronectin - review of its structure and biological-activity," Molecular and Cellular Biochemistry 29(2), pp. 103-128, 1980.

[22] W. Friess, "Collagen - biomaterial for drug delivery," European Journal of Pharmaceutics and Biopharmaceutics 45(2), pp. 113-136, 1998.

[23] S. Ayad, R. P. Boot-Handford, M. J. Humphries, K. E. Kadler, and C. A. Shuttleworth, The Extracellular Matrix Facts Book, Academic Press, New York, 2nd ed., 1998.

[24] P. R. A. Schneider, C. Buhrmann, A. Mobasheri, U. Matis, and M. Shakibaei, "Threedimensional high-density co-culture with primary tenocytes induces tenogenic differentiation in mesenchymal stem cells," Journal of Orthopaedic Research 29(9), pp. 13511360, 2011.

[25] P. Cserjesi, D. Brown, K. L. Ligon, G. E. Lyons, N. G. Copeland, D. J. Gilbert, N. A. Jenkins, and E. N. Olson, "Scleraxis - a basic helix-loop-helix protein that prefigures skeletal formation during mouse embryogenesis," Development 121(4), pp. 1099-1110, 1995.

[26] A. L. Carlberg, R. S. Tuan, and D. J. Hall, "Regulation of scleraxis function by interaction with the bhlh protein e47," Molecular Cell Biology Research Communications 3(2), pp. 82-86, 2000.

[27] S. E. Taylor, A. Vaughan-Thomas, D. N. Clements, G. Pinchbeck, L. C. Macrory, R. K. W. Smith, and P. D. Clegg, "Gene expression markers of tendon fibroblasts in normal and diseased tissue compared to monolayer and three dimensional culture systems," Bmc Musculoskeletal Disorders 10, 2009.

[28] C. L. Mendias, J. P. Gumucio, K. I. Bakhurin, E. B. Lynch, and S. V. Brooks, "Physiological loading of tendons induces scleraxis expression in epitenon fibroblasts," Journal of Orthopaedic Research 30(4), pp. 606-612, 2012.

[29] K. Yamana, H. Wada, Y. Takahashi, H. Sato, Y. Kasahara, and M. Kiyoki, "Molecular cloning and characterization of chm1l, a novel membrane molecule similar to chondromodulin-i," Biochemical and Biophysical Research Communications 280(4), pp. 1101-1106, 2001.

[30] J. Qi, J. M. Dmochowski, A. N. Banes, M. Tsuzaki, D. Bynum, M. Patterson, A. Creighton, S. Gomez, K. Tech, A. Cederlund, and A. J. Banes, "Differential expression 
and cellular localization of novel isoforms of the tendon biomarker tenomodulin," Journal of Applied Physiology 113(6), pp. 861-871, 2012.

[31] V. Kishore, J. A. Uquillas, A. Dubikovsky, M. A. Alshehabat, P. W. Snyder, G. J. Breur, and O. Akkus, "In vivo response to electrochemically aligned collagen bioscaffolds," Journal of Biomedical Materials Research Part B-Applied Biomaterials 100B(2), pp. 400-408, 2012.

[32] Z. Yin, X. Chen, J. L. Chen, W. L. Shen, T. M. H. Nguyen, L. Gao, and H. W. Ouyang, "The regulation of tendon stem cell differentiation by the alignment of nanofibers," Biomaterials 31(8), pp. 2163-2175, 2010.

[33] S. R. Caliari, D. W. Weisgerber, M. A. Ramirez, D. O. Kelkhoff, and B. A. C. Harley, "The influence of collagen-glycosaminoglycan scaffold relative density and microstructural anisotropy on tenocyte bioactivity and transcriptomic stability," Journal of the Mechanical Behavior of Biomedical Materials 11, pp. 27-40, 2012.

[34] G. R. Nakayama, M. C. Caton, M. P. Nova, and Z. Parandoosh, "Assessment of the alamar blue assay for cellular growth and viability in vitro," Journal of Immunological Methods 204(2), pp. 205-208, 1997.

[35] A. D. Mazzocca, D. Chowaniec, M. B. McCarthy, K. Beitzel, M. P. Cote, W. McKinnon, and R. Arciero, "In vitro changes in human tenocyte cultures obtained from proximal biceps tendon: multiple passages result in changes in routine cell markers," Knee Surgery Sports Traumatology Arthroscopy 20(9), pp. 1666-1672, 2012.

[36] R. Schweitzer, J. H. Chyung, L. C. Murtaugh, A. E. Brent, V. Rosen, E. N. Olson, A. Lassar, and C. J. Tabin, "Analysis of the tendon cell fate using scleraxis, a specific marker for tendons and ligaments," Development 128(19), pp. 3855-3866, 2001.

[37] Y. Z. Wang, U. J. Kim, D. J. Blasioli, H. J. Kim, and D. L. Kaplan, "In vitro cartilage tissue engineering with $3 \mathrm{~d}$ porous aqueous-derived silk scaffolds and mesenchymal stem cells," Biomaterials 26(34), pp. 7082-7094, 2005.

[38] V. Kishore, W. Bullock, X. Sun, W. S. Van Dyke, and O. Akkus, "Tenogenic differentiation of human mscs induced by the topography of electrochemically aligned collagen threads," Biomaterials 33(7), pp. 2137-44, 2012. 